\title{
Explicación de la satisfacción en la dirección escolar a partir del desempeño de la función directiva
}

\section{An Explanation of Job Satisfaction in School Principals Based on their Performance of the Position}

Inmaculada Martínez García, Javier Gil Flores

Dpto. Métodos de Investigación y Diagnóstico en Educación. Facultad de Ciencias de la Educación. Universidad de Sevilla

\{inmamartinez; j lores\}@us.es

\section{ORCID: http:///orcid. org/0000-0002-4507-7410; http://orcid. org/0000-0003-0755-4367}

\section{Resumen}

La dirección escolar y el ejercicio del liderazgo pedagógico son vinculantes en la mejora de la calidad de los centros educativos y en su funcionamiento. Es vital que las personas al frente del cargo estén satisfechas con la función de dirección que llevan a cabo, hecho que deriva en una mayor motivación y compromiso con su desempeño profesional. En este trabajo estudiamos la satisfacción laboral de los directores de centros de Educación Secundaria Obligatoria, dada la relevancia del cargo para el buen funcionamiento de las instituciones escolares. Para ello, llevamos a cabo un análisis secundario a partir de datos obtenidos en la última edición del estudio Teaching and Learning International Survey (TALIS) promovido por la Organización para la Cooperación y el Desarrollo Económicos (OCDE en adelante) (OECD, 2014) con el objetivo de explicar la satisfacción en la dirección escolar a partir de variables que hacen referencia al ejercicio de la función directiva. Los resultados muestran mayor satisfacción de la dirección cuando esta hace partícipe a la comunidad educativa de la toma de decisiones, ejerciendo su labor de liderazgo pedagógico y fomentando una cultura de colaboración. Entre las tareas relacionadas con la insatisfacción laboral se encuentran aquellas vinculadas al modelo de gestión burocrático tradicional todavía vigente. Finalmente se ofrecen propuestas orientadas a la mejora de la satisfacción de la dirección basadas en la formación continua a través de itinerarios profesionales de dirección y la formación en competencias relacionadas con el liderazgo distribuido, la creación de espacios de colaboración entre los agentes educativos y la potenciación de las comunidades de aprendizaje.

\section{Palabras Clave}

Satisfacción laboral; directores escolares; enseñanza secundaria

\section{Abstract}

School management and pedagogical leadership are key in the improvement of the quality of schools. It is vital to people in charge to be satisfied with their performance of the position, a fact that leads to a greater motivation and commitment. In this paper we study job satisfaction of principals in secondary schools, given the importance of educational agents for the proper running of schools. To this end, a secondary data analysis is carried out based on data obtained in the latest edition of the Teaching and Learning International Survey (TALIS) study promoted by the OECD (OECD, 2014) with the aim of explaining satisfaction in the school management related to variables associated to the performance of the role. The results show a greater satisfaction of principals when they allow the community to participate in the decision making process, when they perform a distributed leadership and promote a collaborative culture. Among the tasks related to job dissatisfaction are those linked to the traditional bureaucratic management model still prevailing. Based on the results, there have been made some proposals based on the development of professional management paths and training in skills related to distributed leadership, the promotion of collaboration spaces between educational agents and learning communities.

\section{Keywords}

Job Satisfaction; School Principals; Leadership; Secondary Education 


\section{Marco teórico}

La figura de la dirección escolar es clave para la calidad educativa, la innovación docente, el rendimiento del centro y el éxito escolar. En la literatura al respecto, se coincide en afirmar que la dirección escolar y el ejercicio del liderazgo pedagógico desempeñado a través de la misma, son clave en la mejora de la eficacia y en la calidad de las escuelas, así como en la mejora del funcionamiento de los centros (Bolívar, 2010; García, 2014; Rodríguez, Rodríguez, Artíles, Aguiar \& Alemán, 2013; Gómez, 2011; Sarasúa, 2013; Vila, 2015). Por tanto, la dirección escolar es esencial en la revitalización de los centros educativos, ya que ayuda a promover la creación de una cultura propia, aunque se trate de una figura compleja en su funcionamiento. Rodríguez et al. (2013) establecen que motivaciones de tipo altruistas, como la mejora del centro o la satisfacción personal, son las que hacen que un docente decida ejercer la dirección escolar. Este hecho, junto a la relevancia de esta figura en el funcionamiento de los centros, hace necesario que los directores se encuentren satisfechos con las tareas que realizan y con la función de dirección que llevan a cabo en su día a día.

La satisfacción laboral es clave para el buen funcionamiento de las instituciones educativas, convirtiéndose en objeto de estudio en la literatura especializada (Caballero \& Salvador, 2004; Gil, 2017; Mercer, 2010; Ramery \& Pérez, 2016; Tejero \& Fernández, 2009). En este sentido encontramos investigaciones como las de Martínez (2017) que ponen en evidencia que el ambiente laboral y el apoyo de la dirección son variables que influyen en la satisfacción docente. Sin embargo, haciendo referencia a la satisfacción de los directores específicamente, son varios los factores que aparecen asociados a la misma. Por ejemplo, los resultados de algunas investigaciones indican que los directores escolares con mayor nivel de satisfacción son los que cuentan con un nivel alto de preparación para el desempeño del cargo, han realizado tareas formativas previamente, cuentan con más experiencia y más edad (Caballero \& Salvador, 2004; Ramery \& Pérez, 2016). Otros factores asociados a la satisfacción laboral de este colectivo son el control sobre el contexto, la percepción positiva de la calidad del centro y las buenas relaciones dentro y fuera del mismo (Mercer, 2010). Los hallazgos de otros estudios destacan el alto grado de satisfacción de la dirección escolar con su trabajo, en especial con el equipo directivo y la secretaría del mismo (Tejero \& Fernández, 2009).

Sin embargo, para comprender la situación en la que se encuentran los directivos y para poder estudiar formas que favorezcan su nivel de satisfacción y sus condiciones laborales, es necesario tener en cuenta cómo entiende la actual legislación a la dirección escolar y cuáles son las funciones que van asociadas a la misma. De forma tradicional, esta figura estaba asociada a procesos burocráticos y de gestión administrativa, encontrándose alejada de la mejora de los resultados y logro de los centros educativos (Vila, 2015). Sin embargo, es a partir de la Ley Orgánica de Mejora y Calidad Educativa 
(2013) cuando se apuesta por el aumento en la autonomía de los centros y de la figura de la dirección como piezas fundamentales para la mejora de la calidad. Estamos ante lo que Bolívar (2010) denominó "tránsito de un modelo burocrático a una dirección pedagógica" (p. 23). Entre las competencias que desde la legislación son otorgadas a este colectivo, están aquellas relacionadas con el ejercicio de un liderazgo pedagógico y colaborativo: "ejercer la dirección pedagógica, promover la innovación educativa e impulsar planes para la consecución de los objetivos del proyecto educativo del centro", "favorecer la convivencia en el centro, garantizar la mediación en la resolución de los conflictos" o "impulsar la colaboración con las familias, con las instituciones y con organismos que faciliten la relación del centro con el entorno" (p. 46). A partir de esta ley se hace hincapié en tareas que apoyen la cooperación docente y que están relacionadas con el liderazgo pedagógico.

En este proceso de redefinición de la función de la dirección, existen aspectos que pueden funcionar como limitantes y que influyen tanto en el desempeño de la funciones como en el nivel de satisfacción de la dirección. Algunos se relacionan con el hecho de que el cargo se desempeña en un contexto donde la persona al frente de la dirección pertenece al propio equipo docente del centro, aspecto que marca su práctica debido a los posibles sesgos corporativos (Bolívar \& Moreno, 2006). Esta es una de las razones por las que las iniciativas y acciones de los directores se encuentran al servicio de diferentes intereses dentro de la comunidad educativa (Navarro-Corona, 2017). Por otro lado, existen autores que afirman que, a pesar del cambio legislativo, todavía nos encontramos en un modelo de gestión escolar burocrático-administrativista donde estas personas se ven colmadas por tareas asignadas desde la administración (Bolívar, 2016a). Esa realidad dificulta el desempeño efectivo de su rol y genera sobrecarga de trabajo, aspectos que se convierten en estresores y que alejan al colectivo de ejercer sus funciones de una manera satisfactoria (Moral \& Amores, 2016; Tejero \& Fernández, 2010). Se apunta también a que existen otros factores relacionados con la insatisfacción laboral, como la falta de recursos, las relaciones con los compañeros docentes, el reconocimiento percibido, el salario y la falta de comunicación con la administración (Ramery \& Pérez, 2016), así como también la falta de tiempo, el sistema basado en la meritocracia en el que están insertos o el individualismo (Santos, 2000). Por otro lado también encontramos referencias a que existen necesidades formativas en relación a los temas de profesionalización de la dirección y liderazgo (García, 2014; Rodríguez, et al., 2013).

Sin embargo, y a pesar de esta situación, no se puede negar que la dirección escolar en el contexto español es una figura clave en la creación de la cultura del centro y en el ambiente laboral y organizativo que existe en el mismo. Tanto es así que autores como Hernández-Castilla, Murillo e Hidalgo (2017) afirman que "en España, la distinción entre el liderazgo escolar y la dirección escolar es casi inexistente" (p. 500). El director en su desempeño de las tareas se encuentra en construcción de una identidad directiva al mismo tiempo que trabaja en la transformación de la cultura del centro, 
el clima y la eficacia escolar. En este contexto, donde de forma tradicional ha existido un modelo de dirección burocrático-gerencialista (García, 2014), en la actualidad avanzamos hacia un nuevo modelo basado en el liderazgo, donde las personas al frente de la misma pasan a desempeñar un liderazgo pedagógico (Bolívar, 2016b). En este caso el director se convierte en dinamizador de la organización creando un clima de trabajo cohesionado, promocionando un clima de aprendizaje y favoreciendo el trabajo colaborativo (Ritaco \& Bolívar, 2016b; Vila, 2015). Sarasúa (2013) considera que el liderazgo que debe ejercer la dirección va más allá, y debe hacer al profesorado partícipe y protagonista de los procesos de cambio manteniendo y potenciando la unidad del centro. Ello sería posible a través del fomento del sentido de responsabilidad de los docentes, haciendo que estos se sientan identificados con el ideario del centro. En este sentido, el término liderazgo distribuido cobra protagonismo frente al liderazgo transformacional con el que de manera tradicional se identificaba a la dirección escolar.

El rol del director escolar conlleva tareas y responsabilidades que abarcan aspectos organizativos, planificación, gestión de recursos, motivación, trabajo en grupo, dinamización y creación de un buen clima de centro, actuar como representación del mismo, mediación, etc. Por este motivo, desde la literatura encargada del estudio del liderazgo en el contexto escolar, se incide en la necesidad de atender estas tareas desde la perspectiva de la dirección colegiada, pasando al mencionado estilo de liderazgo compartido, participativo y democrático (García, Poblete \& Villa, 2006; Ngunyen, \& Hunter, 2018; Sepúlveda \& Aparicio, 2017) con el objetivo de que los miembros de la comunidad educativa asuman responsabilidades y se sientan parte del funcionamiento del centro (Lara, 2004; Moral \& Amores, 2016; Murillo, 2006). El liderazgo distribuido se caracteriza por que el líder crea rutinas que ayudan a transformar la cultura del centro, contribuyendo a la mejora de la satisfacción laboral de los docentes y del desempeño académico en el alumnado, con una pérdida de protagonismo para las funciones y acciones del líder (Spillaner, 2005). Asimismo, el carácter distribuido implica movilizar a los miembros de la comunidad, aunque en la práctica diaria se requiera la combinación con tareas que los directores solamente puedan desempeñar en solitario (Barroso, 2005; López, García, Oliva, Moreta \& Bellerín, 2014).

Ante esta situación nos planteamos ¿qué es necesario para un liderazgo exitoso en la escuela? La literatura ofrece respuestas estableciendo que los líderes educativos deben diseñar planes de mejora en los que se involucre al contexto educativo y se tenga en cuenta la cultura del centro, así como también se hace hincapié en la capacidad para formar equipos de trabajo colaborativo y para la búsqueda de soluciones a problemas reales (Sepúlveda \& Aparicio, 2017). Autores como HernándezCastilla, Murillo e Hidalgo (2017) también hacen referencia a que, para que un director tenga éxito en la escuela, es necesario que lleve a cabo un liderazgo muy humano, donde se promueva la apertura de la escuela a la comunidad, se establezcan metas conjuntas y se favorezca el desarrollo de las 
personas dentro de la organización, aspectos que se relacionan con la forma de hacer fomentada desde el liderazgo distribuido que venimos comentando.

Un importante aspecto a considerar es cómo hacer de la dirección una figura de liderazgo pedagógico. Se apunta a la necesidad de ofrecer una formación específica y continua para entrenar en competencias relacionadas con el liderazgo y con el desarrollo del mismo a un nivel profesional (Álvarez, 2016; Bolívar, 2010; García \& Solbes, 2016; Gómez, 2011; Mehhdinezhad \& Sardarzahi, 2016; Rodríguez, et al., 2013; Sarasúa, 2013; Vila, 2015). De esta forma se favorecerá la distribución de responsabilidades en el centro. Como práctica de liderazgo exitoso se encuentra el trabajo conjunto con otros centros u organismos, potenciando la creación de comunidades profesionales de aprendizaje (Bolívar, López Yáñez \& Murillo, 2014; Lorenzo, 2012; García \& Caballero, 2015).

En el contexto español, el Real Decreto 894/2014 del 17 de octubre desarrolla las características del curso de formación sobre el ejercicio de la función directiva y se puede observar cómo entre las competencias que se fomentan se encuentran aquellas relacionadas con el liderazgo pedagógico, como son la motivación, las habilidades comunicativas, la gestión de conflictos y la convivencia. Sin embargo, existen algunas competencias que difícilmente pueden adquirirse en procesos formales de formación y se hace necesario pensar en contextos formativos más flexibles (Navarro-Corona, 2017). Un ejemplo en este sentido es el que aporta Gómez (2012), quien ofrece la opción de la tutela como una modalidad formativa valorada de forma muy positiva por las personas al frente de la dirección que la han experimentado. Otros autores como Falcón y Díaz (2007), además de subrayar la importancia formativa para el ejercicio de la dirección, hablan de tres momentos formativos, el previo al acceso, la formación para decidir si acceden al cargo y la formación continua una vez están desempeñando sus funciones directivas.

En el presente trabajo, abordamos el estudio de la satisfacción de los directores escolares. En particular, nos hemos centrado en analizar la relación de la satisfacción laboral de este colectivo con variables relativas a la naturaleza de las tareas realizadas, el modo en que se ejerce el liderazgo en la institución escolar y las limitaciones que condicionan la eficacia en el ejercicio de sus funciones.

\section{Método}

Llevamos a cabo un análisis secundario a partir de datos obtenidos en la última edición del estudio Teaching and Learning International Survey (TALIS) promovido por la OCDE (OECD, 2014), en el que participaron un total de 38 países, incluido España. Además de la edición de 2013, en la que se apoya el presente trabajo, TALIS cuenta con una edición anterior desarrollada en 2008 y en estos momentos se trabaja en la aplicación correspondiente a 2018, en la que nuevamente participa nuestro país. La 
finalidad de TALIS es analizar las características, actuaciones y concepciones del profesorado y de los directores, así como el funcionamiento de las instituciones escolares, con el propósito de obtener una visión sobre la educación que sirva de base para el desarrollo de las políticas educativas. Los países participantes podían optar entre la realización del estudio en los niveles de Educación Primaria y superiores de la Educación Secundaria, o bien en los niveles inferiores de la Educación Secundaria. En el caso de España, el estudio se centró en esta segunda opción, concretándose metodológicamente en la realización de encuestas a profesorado y directores de centros que imparten el nivel CINE-2 de la Clasificación Internacional Normalizada de la Educación, equivalente a Educación Secundaria Obligatoria (ESO) en nuestro sistema educativo.

\subsection{Variables e instrumento}

De acuerdo con el objetivo de explicar la satisfacción en la dirección escolar a partir de variables que hacen referencia al ejercicio de la función directiva, hemos diferenciado entre variables criterio y predictoras. Todas ellas provienen de las respuestas dadas por los directores y directoras al cuestionario aplicado en el estudio TALIS. Este cuestionario constaba de un total de 39 preguntas y fue traducido a las cinco lenguas oficiales del país, para su aplicación en las diferentes comunidades autónomas españolas.

En el presente estudio hemos considerado como variable criterio la satisfacción en el ejercicio de la dirección escolar. Esta variable se ha medido a través de cuatro ítems que formaban parte de la pregunta número 39 del cuestionario respondido por los directores. Su enunciado expresa disfrute y satisfacción con la labor desarrollada en el centro y, en general, con su trabajo, o bien arrepentimiento de haber decidido ser director (la tabla 1, en el apartado de resultados, recoge el enunciado de los ítems). Los directores indicaron su grado de acuerdo en una escala de cuatro puntos, desde total desacuerdo (1) a total acuerdo (4). A partir de estos ítems, construimos un índice de satisfacción laboral de los directores, tal y como se mostrará en el siguiente apartado dedicado al análisis de datos.

Las variables predictoras se corresponden con otros quince ítems del cuestionario, que informan sobre diferentes aspectos del ejercicio de la función directiva (el enunciado de los mismos se muestra en las tablas 3, 4 y 5 incluidas en resultados). Cuatro de estos ítems corresponden a la pregunta número 21 del cuestionario y hacen referencia a tareas desarrolladas por la dirección, ante las cuales los directores debían expresar con qué frecuencia las realizan, usando una escala de cuatro grados: nunca o casi nunca (1), a veces (2), a menudo (3) y muy a menudo (4). Cinco ítems aluden al estilo de liderazgo, recogiendo afirmaciones en relación a la cultura colaborativa y el modo en que se adoptan las decisiones en el centro. Estos ítems se incluyen en la pregunta número 22 y ofrecen cuatro modalidades de respuesta en una escala de acuerdo con cuatro grados, similar a la empleada para 
valorar la satisfacción. Los seis ítems restantes provienen de la pregunta número 26 y van referidos a factores que podrían obstaculizar el desempeño de la dirección escolar. Para estos seis ítems, los directores debían expresar en qué medida tales factores limitan la eficacia en el ejercicio de sus funciones, respondiendo mediante una escala con cuatro modalidades: en absoluto (1), poco (2), en cierta medida (3) y mucho (4).

\subsection{Análisis de datos}

Partimos del análisis descriptivo de los 4 ítems sobre satisfacción laboral de los directores, obteniendo su distribución de frecuencias. A continuación, estos ítems son sometidos a análisis de componentes principales para datos categóricos (categorical principal component analysis: CATPCA). Las puntuaciones individuales obtenidas en el modelo unidimensional vienen expresadas como una variable centrada y reducida, que es tomada como índice de satisfacción laboral en la dirección. Para facilitar su manejo, las puntuaciones en este índice han sido trasladadas a una escala de media 5 y desviación típica 2. Posteriormente, hemos obtenido la distribución de frecuencias para cada uno de los ítems que informan sobre el ejercicio de la función directiva y hemos calculado la correlación de Pearson entre estos y el índice de satisfacción. Teniendo en cuenta que el índice de satisfacción construido presenta un nivel de medida en escala de intervalo, hemos utilizado la correlación de Pearson, a pesar de que las restantes variables presentan solo un nivel ordinal. Seguimos así una práctica habitual en la investigación educativa cuando se analizan medidas ordinales. Respecto a otros coeficientes alternativos, el empleo de la correlación de Pearson supone una infravaloración de la relación entre las variables (Martínez-Abad \& Rodríguez-Conde, 2017). La utilización de la correlación de Pearson supondrá, por tanto, un mayor nivel de exigencia para considerar que existe una relación entre dos variables. Calculadas las correlaciones, aquellos ítems que muestran una correlación significativa han sido considerados conjuntamente en un análisis de regresión por pasos, con el que hemos identificado aquellos que resultan relevantes para explicar la satisfacción de los directores. Finalmente, hemos examinado las medias alcanzadas en el índice de satisfacción en función de las respuestas que los directores y directoras habían dado a cada uno de estos ítems, representándolas a través de diagramas de barras y contrastándolas mediante análisis de la varianza.

\section{Resultados}

\subsection{Satisfacción en la dirección escolar}

Tal y como se recoge en la tabla 1, los directores y directoras escolares muestran un nivel de satisfacción alto en el ejercicio de sus funciones directivas. Más del 95\% de los participantes en 
el estudio está de acuerdo o totalmente de acuerdo con los ítems donde se afirma que disfrutan trabajando en su centro, están satisfechos con su labor y, en conjunto, con su trabajo. Entre estos ítems, destaca el porcentaje elevado de directores (64.5\%) que se muestran totalmente de acuerdo con la afirmación de que disfrutan trabajando en su centro. Además el único ítem de carga negativa, que hace referencia a arrepentirse de haber asumido la dirección, ha suscitado el total desacuerdo del $63.5 \%$ de los participantes, con solo un $12.7 \%$ que se identifica con esta posición.

\begin{tabular}{|c|c|c|c|c|}
\hline Ítem & $\begin{array}{c}\text { Totalmente en } \\
\text { desacuerdo }\end{array}$ & En desacuerdo & De acuerdo & $\begin{array}{c}\text { Totalmente de } \\
\text { acuerdo }\end{array}$ \\
\hline $\begin{array}{c}\text { Me arrepiento de haber decidido } \\
\text { ser director }\end{array}$ & 63.5 & 23.9 & 6.6 & 6.1 \\
\hline $\begin{array}{c}\text { Disfruto trabajando en este centro } \\
\text { Estoy satisfecho con mi labor en } \\
\text { este centro }\end{array}$ & 0.5 & 3.6 & 31.5 & 64.5 \\
\hline $\begin{array}{c}\text { En conjunto, estoy satisfecho con } \\
\text { mi trabajo }\end{array}$ & 0.5 & 3.6 & 57.4 & 39.1 \\
\hline
\end{tabular}

Tabla 1. Estadísticos descriptivos para los ítems sobre satisfacción

Mediante análisis CATPCA reducimos los 4 ítems a una sola dimensión, identificada como índice de satisfacción laboral en la dirección. Con el modelo unidimensional resultante se explica un $62.83 \%$ de la varianza, obteniéndose un elevado alpha de Cronbach cifrado en .803, a pesar de que se cuenta con solo cuatro ítems. Como muestra la tabla 2, los pesos de las variables en valores absolutos oscilan entre .725 y .865. El peso del ítem que hace referencia a arrepentirse de ser director es negativo (-.726). En consecuencia, una puntuación alta en el índice de satisfacción laboral en la dirección implica acuerdo con los ítems que expresan satisfacción y desacuerdo con el que expresa arrepentimiento.

\begin{tabular}{|c|c|}
\hline Ítem & Peso \\
\hline Me arrepiento de haber decidido ser director & -.726 \\
\hline Disfruto trabajando en este centro & .725 \\
\hline Estoy satisfecho con mi labor en este centro & .844 \\
\hline En conjunto, estoy satisfecho con mi trabajo & .865 \\
\hline
\end{tabular}

Tabla 2. Saturaciones en la dimensión 1 para la solución CATPCA

\subsection{Ejercicio de la función directiva y satisfacción en la dirección escolar}

a) Satisfacción y tareas desarrolladas en el desempeño de la dirección.

De acuerdo con las respuestas de directores y directoras (tabla 3), la colaboración con el profesorado para resolver problemas de disciplina es una tarea muy frecuente en los centros. Casi la mitad de los encuestados (48.7\%) afırma realizarla muy a menudo. Destaca también la frecuencia con que se proporciona a padres o tutores información sobre el centro y sobre el rendimiento del alumnado, 
tarea que se realiza a menudo o muy a menudo en el $83.8 \%$ de los casos. No obstante, estas dos tareas frecuentes no son generadoras de satisfacción para los directores, teniendo en cuenta que la correlación con el índice de satisfacción laboral en la dirección es muy baja, con valores que no van más allá del .100. En cambio, la satisfacción guarda cierta relación con la adopción de medidas para apoyar la cooperación docente y con la colaboración con directores de otros centros. Aunque se trata de correlaciones bajas, con un valor $\mathrm{r}=.199$ en ambos casos, la relación es estadísticamente significativa $(p<.01)$, mostrando una cierta tendencia a que quienes más frecuentemente realizan este tipo de tareas registren mayores niveles de satisfacción en la dirección escolar. El apoyo a la cooperación docente y la colaboración con otros directores son tareas frecuentes, aunque no se encuentran entre las más realizadas en los centros (solo el 19.8\% y el 14.2\% de directores respectivamente las desarrolla muy a menudo).

\begin{tabular}{|c|c|c|c|c|c|}
\hline & $\begin{array}{c}\text { Nunca } \\
\text { o casi } \\
\text { nunca }\end{array}$ & $\begin{array}{c}\text { A } \\
\text { veces }\end{array}$ & A menudo & $\begin{array}{c}\text { Muy a } \\
\text { menudo }\end{array}$ & $r$ \\
\hline $\begin{array}{c}\text { He colaborado con los profesores para resolver } \\
\text { problemas de disciplina en el aula }\end{array}$ & 2.0 & 14.7 & 34.5 & 48.7 & .031 \\
\hline $\begin{array}{c}\text { He tomado medidas para apoyar la cooperación } \\
\text { entre los profesores a la hora de desarrollar } \\
\text { nuevas prácticas docentes }\end{array}$ & 2.5 & 38.6 & 39.1 & 19.8 & $.199 * \star$ \\
\hline $\begin{array}{c}\text { He proporcionado a los padres o tutores } \\
\text { información acerca del centro y del rendimiento } \\
\text { de los alumnos }\end{array}$ & 2.0 & 14.2 & 51.3 & 32.5 & .101 \\
\hline He colaborado con directores de otros centros & 5.1 & 46.2 & 34.5 & 14.2 & $.199 * \star$ \\
\hline
\end{tabular}

$\star p<.05 ; * \star p<.01$

Tabla 3. Distribución de frecuencias para las tareas desarrolladas por la dirección y correlaciones con la satisfacción

b) Satisfacción y estilo de liderazgo.

El estilo de liderazgo que caracteriza al ejercicio de la función directiva en los centros que imparten ESO se aproxima en general a un liderazgo distribuido. De acuerdo con los resultados mostrados en la tabla 4, existe una cultura de colaboración en el centro (en el 91.9\% de los casos se da a menudo o muy a menudo) y se ofrecen oportunidades para participar en las decisiones del centro al profesorado y otro personal (98.9\%), a los padres o tutores (91.9\%) y al alumnado (84.2\%). Más de la mitad de los directores y directoras (55.3\%) nunca o casi nunca toman en solitario las decisiones importantes, aunque se registran porcentajes de cierta cuantía para quienes afirman hacerlo a veces (36.5\%) o a menudo (5.6\%). No obstante, la adopción de las decisiones importantes sin contar con otros actores presentes en el centro es el único elemento que no correlaciona con la satisfacción en el ejercicio de la dirección. Resulta más relevante para la satisfacción de directores y directoras el desempeño de un liderazgo que propicia la colaboración y la participación de los distintos estamentos escolares, registrándose para los cuatro ítems implicados correlaciones positivas, aunque de baja intensidad (valores de r comprendidos entre .197 y .333, todos ellos con p<.01). En consecuencia, la satisfacción 
en la dirección tiende a ser mayor cuando se registran mayores niveles de liderazgo distribuido, identificado con la participación de la comunidad escolar en la toma de decisiones y la existencia de una cultura de colaboración en el centro.

\begin{tabular}{|c|c|c|c|c|c|}
\hline & $\begin{array}{l}\text { Totalmente en } \\
\text { desacuerdo }\end{array}$ & $\begin{array}{c}\text { En } \\
\text { desacuerdo }\end{array}$ & $\begin{array}{c}\text { De } \\
\text { acuerdo }\end{array}$ & $\begin{array}{l}\text { Totalmente } \\
\text { de acuerdo }\end{array}$ & r \\
\hline $\begin{array}{c}\text { Este centro ofrece a su personal oportunidades } \\
\text { de participar activamente en las decisiones del } \\
\text { centro }\end{array}$ & - & 1.0 & 49.7 & 49.2 & $.234^{\star \star}$ \\
\hline $\begin{array}{c}\text { Este centro ofrece a los padres o tutores } \\
\text { oportunidades de participar activamente en las } \\
\text { decisiones del centro }\end{array}$ & - & 8.2 & 64.3 & 27.6 & $.214^{\star \star}$ \\
\hline $\begin{array}{c}\text { Este centro ofrece a los alumnos oportunidades } \\
\text { de participar activamente en las decisiones del } \\
\text { centro }\end{array}$ & - & 15.8 & 62.8 & 21.4 & $.197 \star \star$ \\
\hline Tomo yo solo las decisiones importantes & 55.3 & 36.5 & 5.6 & 2.5 & -.128 \\
\hline $\begin{array}{c}\text { Existe una cultura de colaboración en el centro } \\
\text { caracterizada por el apoyo mutuo }\end{array}$ & 1.0 & 7.1 & 57.9 & 34.0 & $.333^{\star \star}$ \\
\hline
\end{tabular}

$\star p<, 05 ; * \star p<, 01$

Tabla 4. Distribución de frecuencias para los ítems relativos al estilo de liderazgo y correlaciones con la satisfacción

c) Satisfacción y factores limitantes de la eficacia en la dirección.

A juicio de los directores y directoras, los factores que en mayor medida limitan la eficacia en la dirección de los centros (tabla 5) son la regulación normativa derivada de las políticas educativas (el $39.1 \%$ de los directores considera que limitan mucho), la sobrecarga que supone el exceso de trabajo y de responsabilidades (33\%), y la falta de presupuesto y recursos (32\%). Por el contrario, no representan factores relevantes la falta de dirección compartida (para el 80.2\% limita poco o en absoluto la eficacia), o la falta de oportunidades y apoyo para su desarrollo profesional (58.9\%). La medida en que los diferentes factores considerados limitan la eficacia en la dirección correlaciona negativamente con la satisfacción expresada por los directores. No obstante, solo parte de estas correlaciones llegan a ser estadísticamente significativas. La importante limitación ocasionada por factores externos, como la falta de recursos y la regulación derivada de las políticas gubernamentales, no se asocia a un mayor o menor grado de satisfacción. En cambio, resultan relevantes para la satisfacción en la dirección el exceso de trabajo y responsabilidades $(r=-.184 ; p<.01)$ y la falta de una dirección compartida con otros miembros de la plantilla del centro $(r=-.273 ; p<.01)$. De este modo, la satisfacción tiende a ser mayor cuando los directores no sienten limitada su eficacia por la sobrecarga de trabajo y responsabilidades, o por la falta de un equipo directivo con el que compartir las tareas de dirección. 


\begin{tabular}{|c|c|c|c|c|c|}
\hline & $\begin{array}{c}\text { En ab- } \\
\text { soluto }\end{array}$ & Poco & $\begin{array}{c}\text { En cierta } \\
\text { medida }\end{array}$ & Mucho & $r$ \\
\hline Falta de presupuesto y de recursos & 4.6 & 10.2 & 53.3 & 32.0 & -.098 \\
\hline Regulación y política del gobierno & 2.5 & 8.1 & 50.3 & 39.1 & -.103 \\
\hline $\begin{array}{c}\text { Falta de participación y apoyo de los padres y } \\
\text { tutores }\end{array}$ & 7.1 & 41.1 & 35.5 & 16.2 & $-.156^{\star}$ \\
\hline $\begin{array}{c}\text { Falta de oportunidades y de apoyo para mi propio } \\
\text { desarrollo profesional }\end{array}$ & 15.2 & 43.7 & 32.0 & 9.1 & $-.180^{\star}$ \\
\hline $\begin{array}{c}\text { Exceso de trabajo y de responsabilidades en mi } \\
\text { trabajo }\end{array}$ & 5.1 & 17.8 & 44.2 & 33.0 & $-.184^{\star \star}$ \\
\hline $\begin{array}{c}\text { Falta de dirección compartida con otros miem- } \\
\text { bros de la plantilla del centro }\end{array}$ & 36.5 & 43.7 & 16.8 & 3.0 & $-.273^{\star \star}$ \\
\hline
\end{tabular}

$\star p<, 05 ; * \star p<, 01$

Tabla 5. Distribución de frecuencias para los factores limitantes de la eficacia en la dirección y correlaciones con la satisfacción

\subsection{Explicación de la satisfacción a partir del ejercicio de la función directiva}

Los resultados anteriores han permitido constatar la existencia de relaciones bivariadas entre la satisfacción expresada por los directores y algunas variables relativas a sus actuaciones, estilo de liderazgo y limitaciones percibidas. A continuación analizamos la importancia explicativa que tienen estas variables cuando son considerados conjuntamente sus efectos sobre la satisfacción. Para ello hemos llevado a cabo una regresión múltiple de la satisfacción sobre el conjunto de ítems que correlacionan significativamente con esta variable. Los ítems han sido introducidos en el modelo por etapas, de tal manera que se han ido incluyendo las variables predictoras más correlacionadas con la satisfacción, hasta que una nueva variable no ha supuesto un incremento significativo de la varianza explicada por el modelo. El modelo final resultante de este proceso explica significativamente la varianza de la satisfacción en la dirección escolar ( $F=14.538 ; p<.001)$, con un valor explicativo R2=.173.

En este modelo únicamente han quedado incluidos tres ítems (tabla 6), referidos a la colaboración en el centro, a compartir las tareas de dirección y a la colaboración con otros centros. Los coeficientes $\beta$ incluidos en la tabla 6 indican la importancia relativa de cada ítem en la explicación de la satisfacción. De acuerdo con los valores obtenidos, la existencia de una cultura colaborativa en el centro es el aspecto más relevante $(\beta=0.281)$ para explicar la satisfacción en la dirección escolar. Atendiendo a los coeficientes no estandarizados, la satisfacción en la dirección escolar se incrementa en 0.898 puntos por cada incremento unitario en el ítem que mide la existencia de una cultura colaborativa en el centro. Este incremento de la satisfacción es de 0.456 en el caso de la colaboración con directores de otros centros, mientras que al aumentar una unidad la limitación de eficacia por falta de dirección compartida se produce un descenso de 0.504 puntos en la satisfacción laboral de los directores. 


\begin{tabular}{|c|c|c|c|c|c|}
\hline & \multicolumn{2}{|c|}{$\begin{array}{c}\text { Coeficientes no } \\
\text { estandarizados }\end{array}$} & $\begin{array}{c}\text { Coefi- } \\
\text { cientes } \\
\text { tipifica- } \\
\text { dos }\end{array}$ & $\mathrm{t}$ & \multirow{2}{*}{ Sig. } \\
\cline { 1 - 4 } (Constante) & $\beta$ & $\begin{array}{c}\text { Error } \\
\text { tipico }\end{array}$ & $\beta$ & & \\
\hline $\begin{array}{c}\text { Existe una cultura de colaboración en el centro } \\
\text { caracterizada por el apoyo mutuo }\end{array}$ & 0.898 & 0.215 & 0.281 & 4.177 & .000 \\
\hline $\begin{array}{c}\text { Falta de dirección compartida con otros } \\
\text { miembros de la plantilla del centro }\end{array}$ & -0.504 & 0.168 & -0.202 & -3.007 & .003 \\
\hline He colaborado con directores de otros centros & 0.456 & 0.164 & 0.182 & 2.782 & .006 \\
\hline
\end{tabular}

Tabla 6. Regresión de la satisfacción sobre variables relativas al ejercicio de la función directiva

Mediante el gráfico 1 hemos representado los niveles de satisfacción en función de la existencia de una cultura de colaboración en el centro, ítem de mayor peso en la explicación de la satisfacción en la dirección escolar. Aquellos directores que están totalmente de acuerdo muestran una media de 5.93 en el índice de satisfacción, mientras los que están en desacuerdo se sitúan en 3.11. La diferencia entre estas medias resulta estadísticamente significativa, de acuerdo con los resultados del análisis de la varianza llevado a cabo $(F=12.34 ; p<.001)$.

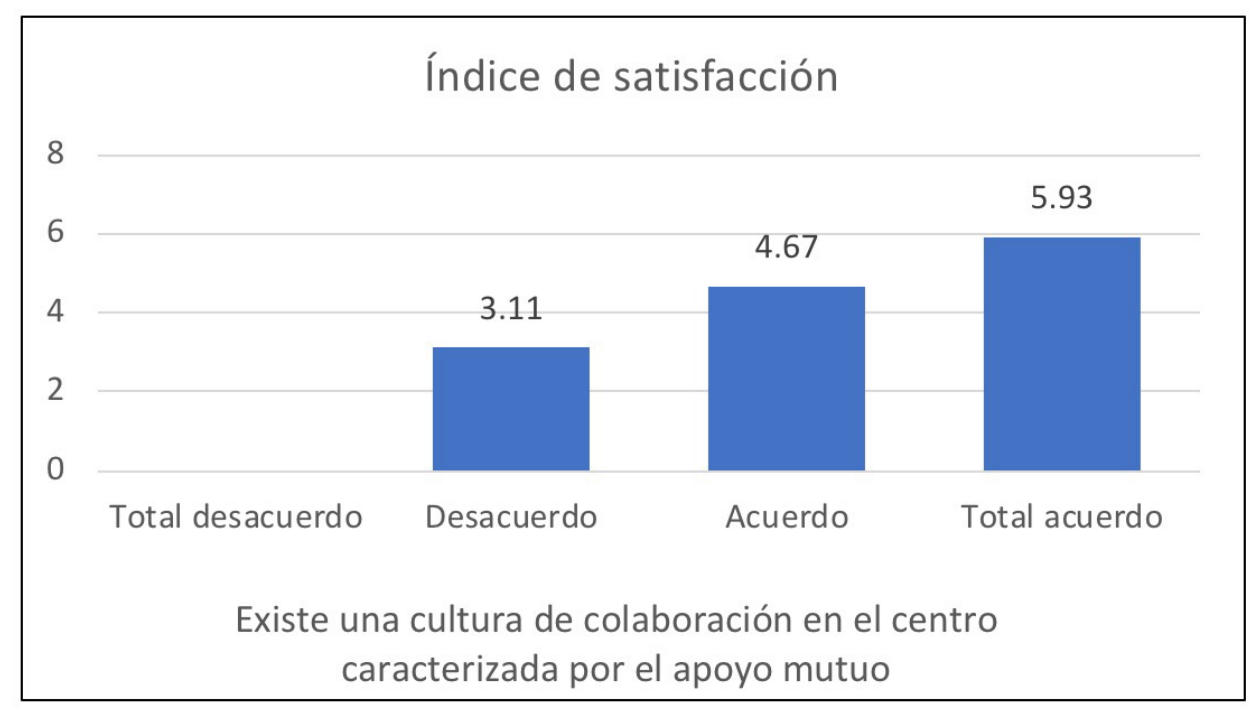

Gráfico 1. Satisfacción en función de la cultura de colaboración en el centro

Cuando la falta de dirección compartida no constituye en absoluto una limitación, el nivel de satisfacción es 5.55, mientras que si se considera una limitación importante, baja hasta 3.25 (ver gráfico 2). Las diferencias observadas en la satisfacción de directores o directoras que discrepan sobre el grado en que la falta de dirección compartida representa una limitación en el ejercicio de sus funciones directivas es estadísticamente significativa ( $F=5.29 ; p=.002)$. 


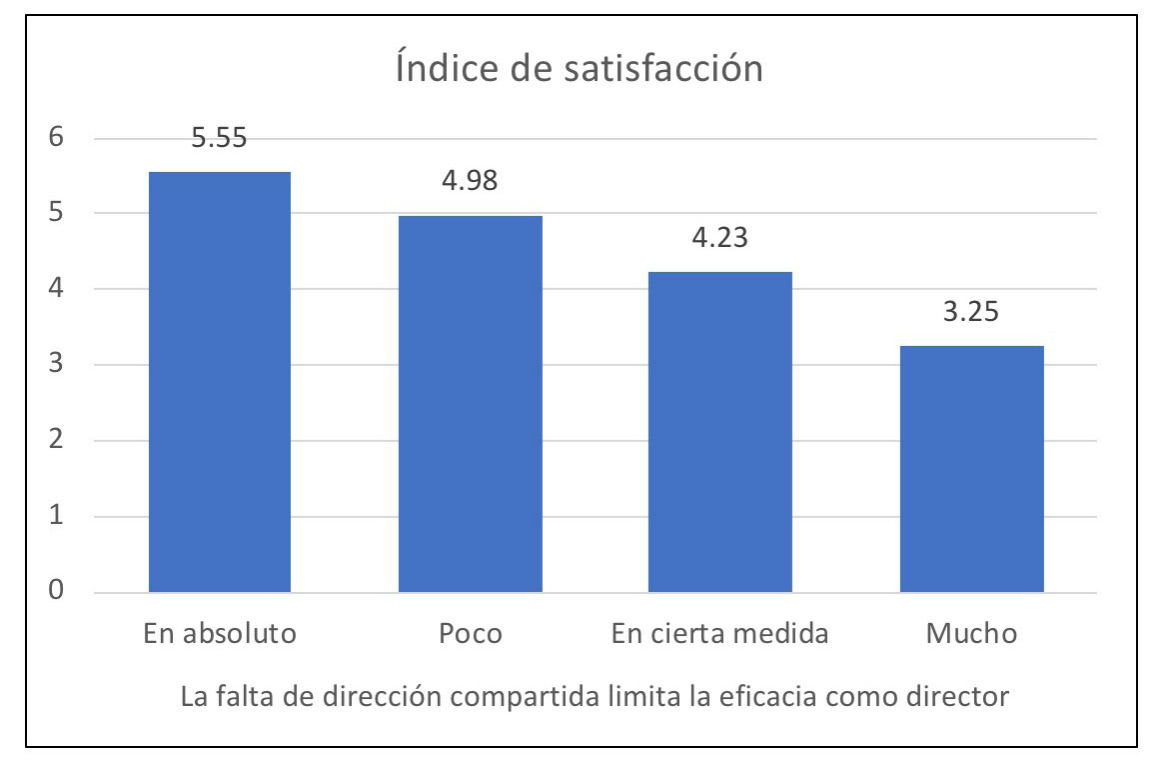

Gráfico 2. Satisfacción en función de la limitación por falta de dirección compartida

Finalmente, la tercera de las variables relevantes en la explicación de la satisfacción en la dirección es la existencia de una coordinación con los directores de otros centros. Como muestra el gráfico 3, cuando esta colaboración no ocurre nunca o casi nunca, el índice de satisfacción se sitúa en 4.43, elevándose hasta 5.99 para los casos en los que esta colaboración se da muy a menudo. La diferencia entre las medias resulta nuevamente significativa ( $F=3.10$ : $p=.028)$.

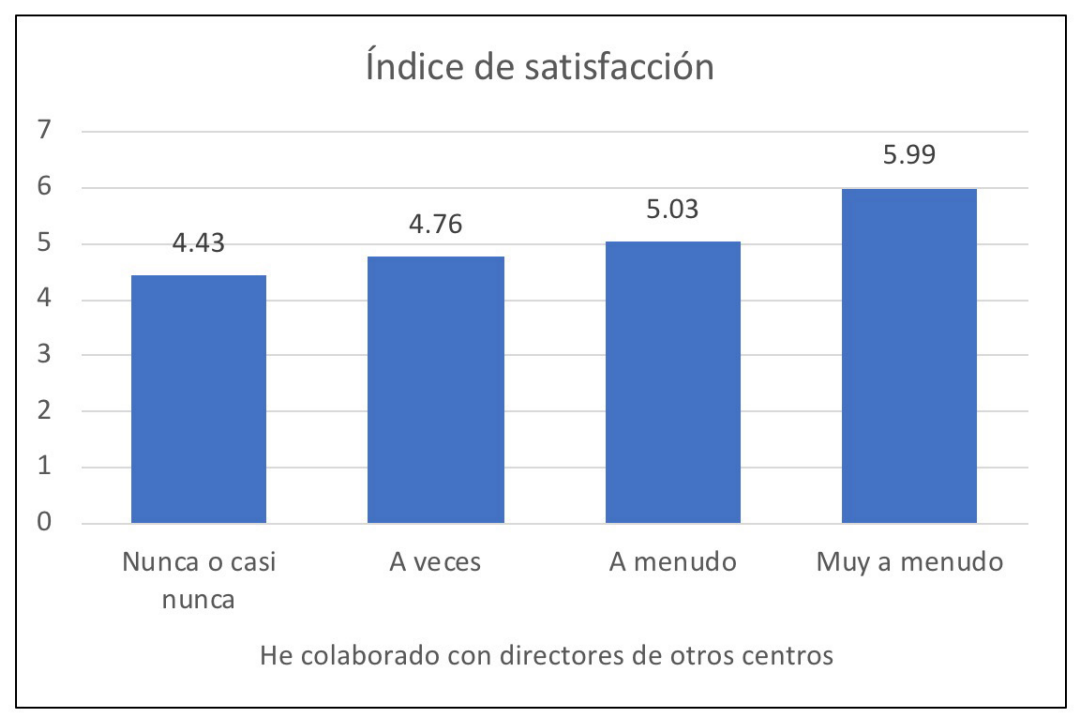

Gráfico 3. Satisfacción en función de la colaboración con la dirección de otros centros

\section{Conclusiones}

En esta investigación hemos constatado que existe un alto grado de satisfacción de los directores escolares con el ejercicio de sus funciones. Los resultados muestran que estos se encuentran muy satisfechos cuando desempeñan tareas asociadas al fomento de la cooperación docente y al trabajo conjunto con directivos de otros centros educativos (Mercer, 2010). Esta satisfacción está relacionada 
con los planteamientos que identifican el liderazgo exitoso con el trabajo junto a otros profesionales y la creación de comunidades de aprendizaje (Lorenzo, 2012). Sin embargo, y a pesar de su relación con el nivel de satisfacción laboral, no son las prácticas más frecuentes en los centros educativos. En consecuencia, de estos resultados cabe derivar la recomendación de fomentar la cooperación dentro de los centros y entre los directores de distintos centros.

El estudio también pone en evidencia que el estilo de liderazgo que se desempeña en la dirección escolar se aproxima al concepto de liderazgo distribuido (García, Poblete \& Villa, 2006; Sepúlveda \& Aparicio, 2017), desde el que se fomenta una cultura de colaboración y se dan oportunidades para participar en la toma de decisiones a otros miembros de la comunidad educativa. Encontramos que más de la mitad de los directores nunca toman en solitario las decisiones importantes, y en caso de hacerlo no se sienten satisfechos por ello, encontrándose más satisfechos cuando llevan a cabo un liderazgo que propicia la cooperación y participación entre los estamentos escolares (Ritaco \& Bolívar, 2016; Vila, 2015).

Haciendo referencia a los factores que aparecen como limitantes en la eficacia de la dirección de centros y que son motivo de insatisfacción laboral, están aquellos derivados de las regulaciones normativas, la sobrecarga de trabajo y responsabilidades, aspectos que se relacionan con el modelo de gestión burocrático todavía vigente comentado por Bolívar (2016b). Asimismo, se valoran también otras limitaciones externas como la falta de recursos y de presupuesto, factores relacionados con la insatisfacción laboral por autores como Ramery y Pérez (2016).

De manera general podemos decir que la dirección se siente más satisfecha cuando toma decisiones de forma colaborativa y hace partícipes a los demás agentes educativos, creando una cultura de centro, fomentando un buen ambiente de trabajo y ejerciendo así su labor de liderazgo pedagógico.

Los resultados de esta investigación podrían resultar de utilidad para el establecimiento de propuestas orientadas hacia la mejora de la formación ofrecida a la dirección escolar y para el fomento de espacios en los que el liderazgo distribuido se haga viable, con las consiguientes repercusiones positivas en el funcionamiento de las instituciones escolares. Estas propuestas se organizan en tres áreas de trabajo a desarrollar de manera conjunta, que expondremos seguidamente.

Haciendo referencia a los aspectos formativos y partiendo de la idea de que el desempeño efectivo del rol y la formación previa se relaciona con la satisfacción laboral (Salvador, 2004; Ramery \& Eufasio, 2016), se plantea la necesidad de ofrecer una formación continua que contemple pautas para entrenar a los directores en competencias relacionadas con el liderazgo a nivel profesional (Álvarez, 2016; García \& Solbes, 2016; Mehhdinezhad \& Sardarzahi, 2016). Esta debe ir más allá de la ofrecida a través de los cursos de formación desarrollados en el Real Decreto 894/2014 del 17 
de octubre, y debe poner el foco en la práctica del liderazgo distribuido y la dirección colegiada. Tomando en consideración la posibilidad de ofrecer contextos formativos flexibles (Navarro-Corona, 2017) adaptados a la disponibilidad y necesidades de los directivos, se sugiere contemplar la opción apuntada por Álvarez (2016) sobre la creación de un itinerario profesional de dirección que abarque una formación continua en el tiempo atendiendo a las necesidades que surjan, idea desarrollada también por Falcón y Díaz (2007). Esta formación continua podría ir acompañada de un sistema de tutorización o mentorización en la línea de la propuesta de la tutela directiva que ofrece Gómez (2012) y cuyos resultados son valorados positivamente, contando los directivos con un acompañamiento de personas que han desempeñado previamente el cargo.

En segundo lugar, de acuerdo con el ejercicio de un liderazgo distribuido, se propone la creación de espacios de colaboración tanto físicos como temporales entre agentes educativos, donde las voces de los líderes de carácter informal cobran relevancia (Spillaner, 2005). Esto ayudarían a que los miembros de la comunidad educativa se sientan implicados en la toma de decisiones y en el funcionamiento del centro (Lara, 2004; Murillo, 2006), además de favorecer la distribución de la sobrecarga de trabajo identificada como un estresor principal (Moral \& Amores, 2016) y favorecer el trabajo colaborativo, ya que el director funcionaría como dinamizador del centro (Ritaco \& Bolívar, 2016).

La tercera propuesta se relaciona con la potenciación de comunidades profesionales de aprendizaje (Bolívar, López Yáñez \& Murillo, 2014; Lorenzo, 2012; García \& Caballero, 2015) fomentando el desempeño de un liderazgo pedagógico. Esta sería producto del fortalecimiento de la colaboración con el contexto donde se sitúa el centro, y con otros centros u organismos atendiendo a las líneas ofrecidas por Bolívar, López Yáñez y Murillo (2014), Lorenzo (2012) y García y Caballero, (2015). Para ello se deben crear espacios y tiempos en los que los directivos de diferentes centros puedan reunirse para la planificación de acciones siguiendo la idea de liderazgo compartido. Esto podría contribuir tanto a la mejora de la calidad del centro como de la comunidad en la que este se sitúa.

El trabajo que aquí hemos presentado se beneficia del rigor metodológico que caracteriza al estudio TALIS. En este sentido, una fortaleza se encuentra en la utilización de una muestra de directores y directoras de todas las comunidades autónomas españolas, que permite enmarcar los resultados en el contexto nacional y no restringirlos a una región concreta. Sin embargo, es necesario tener en cuenta algunas limitaciones, también derivadas del diseño adoptado. Por ejemplo la muestra, aun siendo heterogénea, no alcanza un tamaño lo suficientemente grande para generalizar, con un elevado nivel de confianza, los resultados a la población de directores escolares. Además, la información recogida se apoya en técnicas de autoinforme y podría, por tanto, verse afectada por la subjetividad de los informantes. Por otra parte, el enfoque correlacional asumido nos lleva a constatar relaciones entre 
variables y valorar en qué medida contribuyen a explicar la satisfacción en la dirección los diferentes factores relativos al desempeño de la función directiva, pero no permite establecer relaciones causales, que identifiquen razones por las que los directoras y directores escolares se muestran en mayor o menor medida satisfechos. Saliendo al paso de esta última limitación, en futuros trabajos sería interesante la adopción de diseños cuasi-experimentales, para constatar los cambios producidos en la satisfacción de los directores tras el desarrollo de estrategias basadas en propuestas como las que antes hemos formulado.

\section{Referencias}

Álvarez, M. (2016). Profesionalizar la dirección escolar. Organización y gestión educativa: Revista del Fórum Europeo de Administradores de la Educación, 24(1), 20-25.

Barroso, J. (2005). Liderazgo y autonomía de los centros educativos. Revista Española de Pedagogía, 63(232), 423-441.

Bolívar, A. (2010). El liderazgo educativo y su papel en la mejora: Una revisión actual de sus posibilidades y limitaciones. Psicoperspectivas, 9(2), 9-33. doi:https://doi.org/10.5027/ psicoperspectivas-Vol9-Issue2-fulltext-112

Bolívar, A. (2016a). Evaluar el liderazgo pedagógico de la dirección escolar. Revisión de enfoques e instrumentos. Revista iberoamericana de Evaluación Educativa, 8, 15-39. doi:https:// doi.org/10.15366/riee

Bolívar, A. (2016b). Conjugar el liderazgo pedagógico de la dirección escolar y su profesionalización en el contexto español. Organización y gestión educativa: Revista del Fórum Europeo de Administradores de la Educación, 24(1), 26-29.

Bolívar, A., López Yáñez, J. y Murillo, F. J. (2014). Liderazgo en las instituciones educativas. Una revisión de líneas de investigación. Revista Fuentes, 14, 15-60.

Bolívar, A. y Moreno, J. M. (2006). Between transaction and transformation: The role of school principals as education leaders in Spain. Journal of Educational Change, 7(1-2), 19-31. doi:https://doi.org/10.1007/s10833-006-0010-7

Caballero, J. y Salvador, F. (2004). Satisfacción e insatisfacción de los directores escolares. XXI. Revista de Educación, 333, 363-384.

Falcón, A. y Díaz, L. (2007). Variables que predicen la satisfacción y la motivación de los directores de organizaciones educativas. Avances de Supervisión Educativa, 6 (1-46). 
García M. y Caballero, K. (2015). ¿Qué prácticas eficaces de liderazgo desarrollan los directivos andaluces en sus escuelas? Revista Iberoamericana de Evaluación Educativa, 8(2), 129147. doi:https://doi.org/10.15366/riee

García, M. A. (2014). La evolución de la función directiva en España a través de la legislación educativa: un recorrido por más de un siglo de historia. Aula de Encuentro, 1(16), 139-155.

García, A., Poblete, M. y Villa, A. (2006). La función directiva: un problema sin resolver. Tres décadas e formación, investigación y acción. XXI. Revista de educación, 8, 13-34.

García, S. y Solbes, J. R. (2016). Los equipos directivos en la formación profesional española: una propuesta de formación continua. Revista Iberoamericana de Educación, 70, 161-182.

Gil, J. (2017). Características personales y de los centros educativos en la explicación de la satisfacción laboral del profesorado. Revista de Psicodidáctica, 22(1), 16-22. doi: 10.1016/ S1136-1034(17)30039-4

Gómez, A. M. (2011). La descentralización de la formación inicial para la dirección escolar en España. Profesorado. Revista de currículum y formación del profesorado, 15(2), 199-217.

Gómez, A. M. (2012). La sostenibilidad del liderazgo escolar en Andalucía a través de la modalidad formativa de tutela. Educatio Siglo XXI, 30(2), 423-444.

Hernández-Castilla, R., Murillo, F. J. y Hidalgo, N. (2017). Lecciones Aprendidas del Estudio del Liderazgo Escolar Exitoso. Los casos de España en el Proyecto Internacional ISSPP. Revista de Investigación Educativa, 35(2), 499-518. doi:https://doi.org/10.6018/rie.35.2.279241

Lara, F. (2004). La escuela como compromiso. Madrid: Ed. Popular.

Ley Orgánica 8/2013 de 9 de diciembre para la Mejora de la Calidad Educativa. (Boletín Oficial del Estado, de 10 de diciembre de 2013).

López, J., García, E., Oliva, N., Moreta, B. y Bellerín, A. (2014). El liderazgo escolar a través del análisis de la actividad diaria de los directores. REICE. Revista Iberoamericana sobre Calidad, Eficacia y Cambio en Educación, 12(5), 61-78.

Lorenzo, M. (2012). Las comunidades de liderazgo de centros educativos. Revista Educar, 48(1), 9-21. http://www.redalyc.org/html/3421/342130838002/

Martínez, C. (2017). La incidencia del liderazgo y el clima escolar en la satisfacción laboral de los docentes en América Latina. Archivos analíticos de políticas educativas, 25(80), 1-22. doi: https://doi.org/10.14507/epaa.25.2851 
Martínez-Abad, F. y Rodríguez-Conde, M. J. (2017). Comportamiento de las correlaciones producto-momento y tetracórica-policórica en escalas ordinales: un estudio de simulación. RELIEVE - Revista Electrónica de Investigación y Evaluación Educativa, 23(2). doi:https://doi. org/10.7203/relieve.23.2.9476

Mehhdinezhad, V. y Sardarzahi, Z. (2016). Leadership behaviors and its relation with principals' management experience. NAER: Journal of new approaches in educational research, 5(1), 11-16. doi: https://doi.org/10.7821/naer.2016.1.133

Mercer, D. (2010). Job satisfaction and the Secondary Headteacher: the creation of a model of job satisfaction. School Leadership and Management, 17(1), 57-68. doi:https://doi. org/10.1080/13632439770168

Moral, C. y Amores, F. J. (2016). Liderazgo distribuido y capacidad de mejora en centros de educación secundaria. Estudios sobre Educación, 30, 115-143. doi:https://doi. org/10.15581/004.30.115-143

Murillo, J. (2006). Una dirección escolar para el cambio: del liderazgo transformacional al liderazgo distribuido. REICE. Revista Electrónica Iberoamericana sobre Calidad, Eficacia y Cambio en Educación, 4(4), 12-24.

Navarro-Corona, C. (2017). Estrategias de aprendizaje de los directores escolares en la sociedad del conocimiento. Education in the Knowledge Society, 18(4), 97-112. doi:https://doi. org/10.14201/eks201718497112

Ngunyen, T. D. y Hunter, S. (2018). Towards an understanding of dynamics among teachers, teacher leaders, and administrators in a teacher-led school reform. Journal of Educational Change, 18, 1-27. doi:https://doi.org/10.1007/s10833-017-9316-x

OECD. (2014). TALIS 2013 Results: An International Perspective on Teaching and Learning. doi:https://doi.org/10.1787/9789264196261-en

Ramery, E. y Pérez, E. (2016). Comparación de la satisfacción laboral del director escolar y los docentes. Revista Electrónica de Investigación y Docencia, 15(1), 85-100. doi: https:// doi.org/10.17561/reid.v0i15.2797

Real Decreto 894/2014 del 17 de octubre por el que se desarrollan las características del curso de formación sobre el desarrollo de la función directiva (Boletín Oficial del Estado, 270, de 7 de noviembre de 2014). 
Ritaco, M. y Bolívar, A. (2016). Impacto del modelo español de dirección escolar en la identidad profesional de los líderes escolares. Archivos Analíticos de Políticas Educativas, 24(119), 1-34. doi:https://doi.org/10.14507/epaa.24.2512

Rodríguez, J., Rodríguez, A. J., Artíles, J., Aguiar, M. V. y Alemán, J. A. (2013). El acceso a la dirección escolar: dificultades y necesidades. Educar, 49(1), 105-125. doi:https://doi. org/10.5565/rev/educar.13

Santos, M. A. (2000). Dirección escolar e innovación educativa. Revista de Educación, 2, 61-76.

Sarasúa Ortega, A. (2013). La dirección escolar, lunes y sombras. Padres y Maestros, 350, 4144.

Sepúlveda, F. y Aparicio, C. (2017). El desafío de los directores de escuelas chilenas: liderando a partir de un enfoque instruccional hacia un enfoque distribuido. Revista Gestión de la Educación, 7(2), 1-19. doi: https://doi.org/10.15517/rge.v7i2.30599

Spillaner, J. P. (2005). Distributed Leadership. The Educational Forum, 69(2), 143-150. doi:https://doi.org/10.1080/00131720508984678

Tejero, C. y Fernández, M. J. (2009). Medición de la satisfacción laboral en la dirección escolar. RELIEVE, 15(2), 1-16. doi:https://doi.org/10.7203/relieve.15.2.4160

Tejero, C. y Fernández, M. J. (2010). Estrés laboral y dirección escolar: escala de medición y jerarquía de estresores. Bordón. Revista de Pedagogía, 62(1), 123-137.

Vila, A. (2015). Importancia e impacto del liderazgo educativo. Padres y maestros, 361, 6-11. doi:https://doi.org/10.14422/pym.i361.y2015.001 